\title{
Möglichkeiten der vaskularisierten Knochentransplantationen bei Defektpseudarthrosen an ausgewählten Beispielen der oberen Extremität
}

Saskia Carolin Evers, Adrien Daigeler, Theodora Wahler

\section{Einleitung}

Die Pseudarthrose wird in der Literatur als eine ausbleibende Knochenbruchheilung mindestens 6 Monate nach Trauma definiert. Dabei liegt die Inzidenz zwischen 1 und $5 \%$, abhängig von der Frakturlokalisation. Begünstigende Faktoren für das Entstehen einer Pseudarthrose sind u.a. Infekte, offene sowie instabile bzw. nicht ausreichend stabilisierte Frakturen [1]. Insbesondere eine Minderdurchblutung des Knochens, bspw. begünstigt durch Rauchen, hohes Alter des Patienten oder ein Diabetes mellitus, steigert das Risiko einer ausbleibenden Knochenbruchheilung [2]. Klinisch fällt eine Pseudarthrose oft durch von dem Patienten geäußerte persistierende Schmerzen ohne Besserungstendenz auf. Den Goldstandard zur Diagnostik stellen zunächst das konventionelle Röntgen und als Erweiterung die Computertomografie dar. Eine Kernspintomografie kann Aufschlüsse über eine Minderdurchblutung des Knochens oder einen Infekt geben [1]. Pseudarthrosen können anhand ihrer Morphologie in hypertrophe Pseudarthrosen, atrophe Pseudarthrosen, Defektpseudarthrosen und Infektpseudarthrosen unterschieden werden. Ein konservativer Therapieversuch ist in der Frühphase bei ausreichender Stabilität möglich. Eine operative Therapie sollte in Anpassung an die Pathophysiologie und Morphologie der Pseudarthrose ausgewählt werden. Liegt z. B. eine nicht ausreichende Stabilisierung der Fraktur vor, sollte ein entsprechendes Osteosyntheseverfahren gewählt werden. Eine Minderdurchblutung kann zunächst durch das Anfrischen der Frakturenden, durch Spongiosaplastik, aber bspw. auch durch die Verwendung eines vaskularisierten Knochenspans verbessert werden [2].

Dieser Artikel soll anhand ausgewählter Beispiele der oberen Extremität einen kurzen Überblick über verschiedene Methoden der vaskularisierten Knochentransplantation bei Defektspeudarthrosen bieten.

\section{Pseudarthrosen des Unterarmes}

\section{Einleitung}

Die distale Radiusfraktur gilt als die häufigste Fraktur des Menschen. Dabei zeigen sich in der Häufigkeitsverteilung 2 Altersgipfel. Der 1. Gipfel liegt bei Kindern und Jugendlichen. Der 2. hingegen bei Patienten über 50 Jahren. Ty- pischerweise entsteht eine distale Radiusfraktur des Extensionstyps nach Sturz auf die ausgestreckte dorsal extendierte Hand [3]. In ca. 5-10\% der Fälle tritt zusätzlich zu der distalen Radiusfraktur eine Fraktur der distalen Ulna als Begleitverletzung auf [4]. Die winkelstabile palmare Plattenosteosynthese ist im westeuropäischen Raum sowie den USA das aktuell gängigste operative Verfahren zur Therapie einer distalen Radiusfraktur. Eine ausbleibende Heilung im Sinne einer Pseudarthrose ist in diesem Bereich selten. Arora et al. beschrieben bei 112 Patienten, die im Mittel 15 Monate nach palmarer winkelstabiler Plattenosteosynthese nachuntersucht wurden, in lediglich 4\% eine verzögerte Knochenbruchheilung [5]. Die Pseudarthrosenrate bei Frakturen der Ulna liegt mit ca. 7\% etwas höher [2].

Schaftfrakturen am Unterarm können beide Unterarmknochen betreffen oder isoliert auftreten. Dabei sind beim Erwachsenen häufig direkte Traumata mit Schlag auf den Arm (Parierfraktur), Anpralltraumen im Rahmen von Verkehrsunfällen oder ein Sturz aus großer Höhe ursächlich [6]. In einer retrospektiven Untersuchung von Marcheix et al. wurden die prä- und postoperativen Komplikationen bei 131 Unterarmfrakturen, die mittels Plattenosteosynthese versorgt wurden, ausgewertet. Dabei zeigte sich in 6,9\% der Fälle eine ausbleibende knöcherne Heilung. Dieses Ergebnis korrelierte mit den in der Literatur angegebenen Pseudarthrosenraten von 2-10\% [7].

Pseudarthrosen am Unterarm gehen üblicherweise mit Schmerzen sowie einer eingeschränkten Beweglichkeit des Handgelenks oder auch des Ellenbogens einher und bedürfen daher einer chirurgischen Intervention. Ergibt sich durch den Defekt eine Verschiebung der Relation zwischen den Längen von Radius und Ulna, kann ein Gelenkverschleiß im distalen Radioulnargelenk mit einer schmerzhaften Unterarmumwendbewegung resultieren [8].

\section{Die freie vaskularisierte Fibula}

Die freie Fibulatransplantation wurde erstmals von Huntington et al. im Jahre 1905 publiziert, der mittels eines nicht vaskularisierten Fibulaspans einen Tibiadefekt bei chronischer Osteomyelitis eines Kindes überbrücken konnte [9]. Die erfolgreiche Transplantation einer freien, vaskularisierten Fibula wurde schließlich 1975 von Taylor et al. beschrieben [10]. Der von Taylor beschriebene dorsale Zugang wurde im Folgenden von Gilbert im Sinne 
eines lateralen Zuganges modifiziert [11]. Im Jahr 1979 konnte von Chen und Yan eine erfolgreiche osteokutane Fibulatransplantation bei einer traumatisch bedingten Defektverletzung des Radius und ausgeprägten Narbenkonfigurationen am Unterarm durchgeführt werden [12]. Somit bestehen neben der freien vaskularisierten bzw. nicht vaskularisierten Fibulatransplantation auch die Möglichkeiten eines osteokutanen bzw. osteomuskulären Lappens.

Es gibt 2 Hauptindikationen zur Verwendung einer freien vaskularisierten Fibulatransplantation: die Pseudarthrose oder ein durch ein Trauma oder einen Tumor bedingten Knochendefekt [13]. Dabei gilt inzwischen die freie, vaskularisierte Fibulatransplantation aufgrund der Struktur und Form der Fibula als das am häufigsten verwendete Knochentransplantat bei Defekten im diaphysären Bereich langer Röhrenknochen ( $\boldsymbol{A}$ Abb. $\mathbf{1}$ ). Es kann ein Segment von bis zu ca. $28 \mathrm{~cm}$ Länge verwendet werden [8]. Kürzere Defekte, auch von beiden Unterarmknochen, können durch eine gedoppelte vaskularisierte freie Fibula im Sinne einer „double-barrel fibula“ überbrückt werden [14]. Durch diese zusätzliche Säule kann eine höhere Stabilität erreicht werden. Eine andere Möglichkeit, mehr Stabilität bei längeren Knochendefekten zu erreichen, ist das Einbringen eines autologen vaskularisierten Knochens (z. B. freie Fibula) in ein knöchernes Allograft im Sinne der sog. „Capanna-Technik“ [15].

Die endostale Durchblutung der Fibula erfolgt durch die A. nutritia der A. fibularis [10]. Dabei liegt die Eintrittsstelle des Gefäßes in den Knochen meist in der oberen Hälfte des mittleren Drittels der Fibula, ca. 14-19 cm distal des Fibulakopfes $[13,16]$. Der Gefäßpedikel hat eine Länge von $6-8 \mathrm{~cm}$ und einen Durchmesser von 1,5$3 \mathrm{~mm}$. Es verlaufen üblicherweise 2 Begleitvenen mit der Arterie [8]. Zusätzlich versorgen periostale Äste der A. fibularis und A. tibialis anterior die Diaphyse. Der Fibulakopf wird von den anterioren und posterioren Ästen der A. tibialis anterior versorgt [13].

Der Fibulakopf artikuliert mit der lateralen Tibia und kann zur Rekonstruktion einer Gelenkfläche verwendet werden [13]. Dabei ist jedoch auf den N. peroneus zu achten, der distal des Fibulakopfes herum verläuft [8]. Das distale Ende der Fibula bildet das obere Sprunggelenk. Um die Stabilität des oberen Sprunggelenkes nicht zu gefährden, sollten daher die distalen $6 \mathrm{~cm}$ der Fibula belassen werden [13]. Die in der Literatur beschriebenen Komplikationsraten an der Hebestelle variieren zwischen 7 und $35 \%[17]$.

\section{Der vaskularisierte kortikoperiostale Lappen der medialen Femurkondyle}

Es sind zahlreiche Spenderregionen für einen periostalen Lappen in der Literatur beschrieben. Hierzu zählen u.a. Klavikula, Skapula, distaler Humerus, Radius und Ulna,
Beckenkamm, Femur sowie Tibia und Fibula. Zu den Vorteilen des periostalen Lappens zählt, dass die dünne Membran mit ihrem osteogenen Potenzial gut an verschiedene Defekte anmodelliert werden kann [18]. Im Falle eines langen Röhrenknochens bietet sich eine Umwickelung des Knochens mit dem periostalen Lappen im Sinne eines „wrap-around“-Lappens an.

Im Bereich der oberen Extremität hat sich der kortikoperiostale Lappen des distalen, medialen Femurs bewährt [8]. Dieser wurde 1991 erstmalig von Sakai et al. beschrieben [19]. Dabei ist der Vorteil des kortikoperiostalen Lappens, dass im Gegensatz zu einem rein periostalen Lappen die innerste zellreiche Schicht, das Stratum osteogenicum, intakt gelassen wird [20].

Die Gefäßversorgung erfolgt über die A. genus descendens bzw. die A. superior medialis genus der A. femoralis. Dabei misst der Pedikel der A. genus descendens meist eine Länge von $7 \mathrm{~cm}$ bei einem Gefäßdurchmesser von ca. $2 \mathrm{~mm}$. Ist diese Arterie nicht vorhanden oder zu klein, kann die A. superior medialis genus verwendet werden. Dabei kann ein periostaler Lappen mit einer kortikalen Schicht in einer Länge von bis zu ca. $8 \mathrm{~cm}$ gehoben werden, der quer zur Überbrückung eines knöchernen Defekts bis $2 \mathrm{~cm}$ dienen kann [8].

Trotz guter Ergebnisse sind bislang nur wenige Fälle beschrieben [20-23].

\section{Der freie mediale bzw. laterale osteokutane Paraskapular- oder Skapularlappen}

Im Bereich der Skapula haben sich verschiedene Lappen zur Defektdeckung etabliert. Je nach Spendergefäß wird in den Skapular- (A. cutaneus scapularis aus der A. circumflexa scapulae) oder den Paraskapularlappen (A. cutaneus parascapularis aus der A. circumflexa scapulae) unterschieden ( $\bullet$ Abb. 2 ).

Der fasziokutane Skapularlappen wurde erstmalig durch dos Santos 1980, der Paraskapularlappen durch Nassif et al. 1982 beschrieben. Von Stokes et al. sowie Koshima und Soeda wurden beide Lappen als gemeinsam an einem Gefäßstiel gehobener Lappen beschrieben [24, 25]. Neben dem fasziokutanen Lappen ist auch der osteokutane Lappen möglich. Teot et al. beschrieben 1981 erstmalig den freien vaskularisierten Knochentransfer der lateralen Skapula [26]. Heutzutage werden üblicherweise der laterale oder der mediale Rand der Skapula verwendet. Der Angulus inferior der Skapula wird nur selten verwendet [27].

Der dickste Teil der Skapula, der laterale Rand, wird durch die A. circumflexa scapulae, einen Gefäßast der A. subscapularis, versorgt. Der Angulus inferior wird über ein Gefäßgeflecht aus 3 Arterien versorgt: den absteigenden Ast der A. circumflexa scapulae, den transversalen Ast 

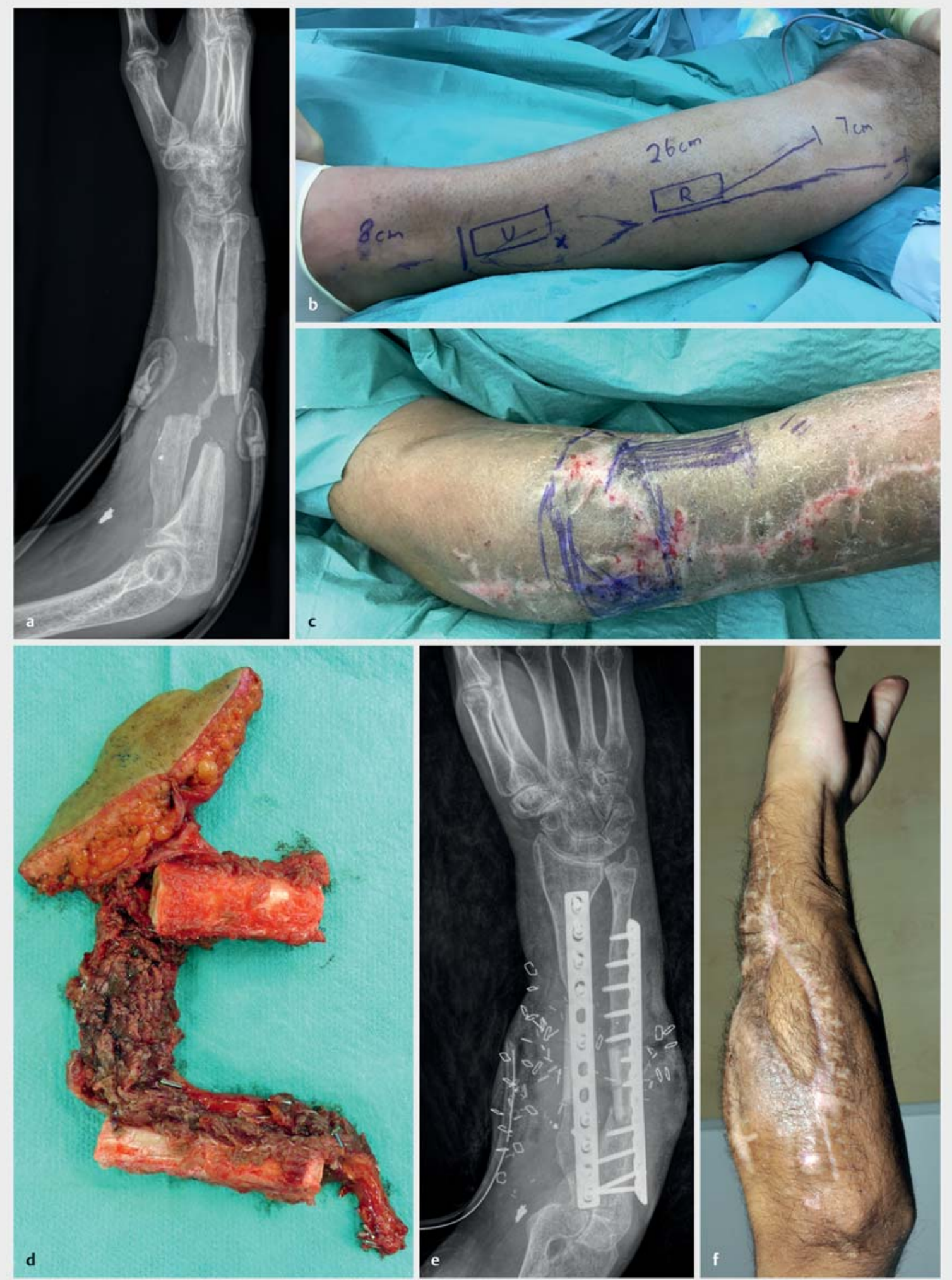

- Abb. 1 a Radiologisches Bild einer Defektpseudarthrose des Unterarmes bei einem 26-jährigen Patienten. b, c Intraoperative Planung des benötigten Knochenspans. d Gefäßgestielter Femurspan mit Hautinsel. e, f Radiologisches und klinisches Ergebnis nach der Rekonstruktion. 


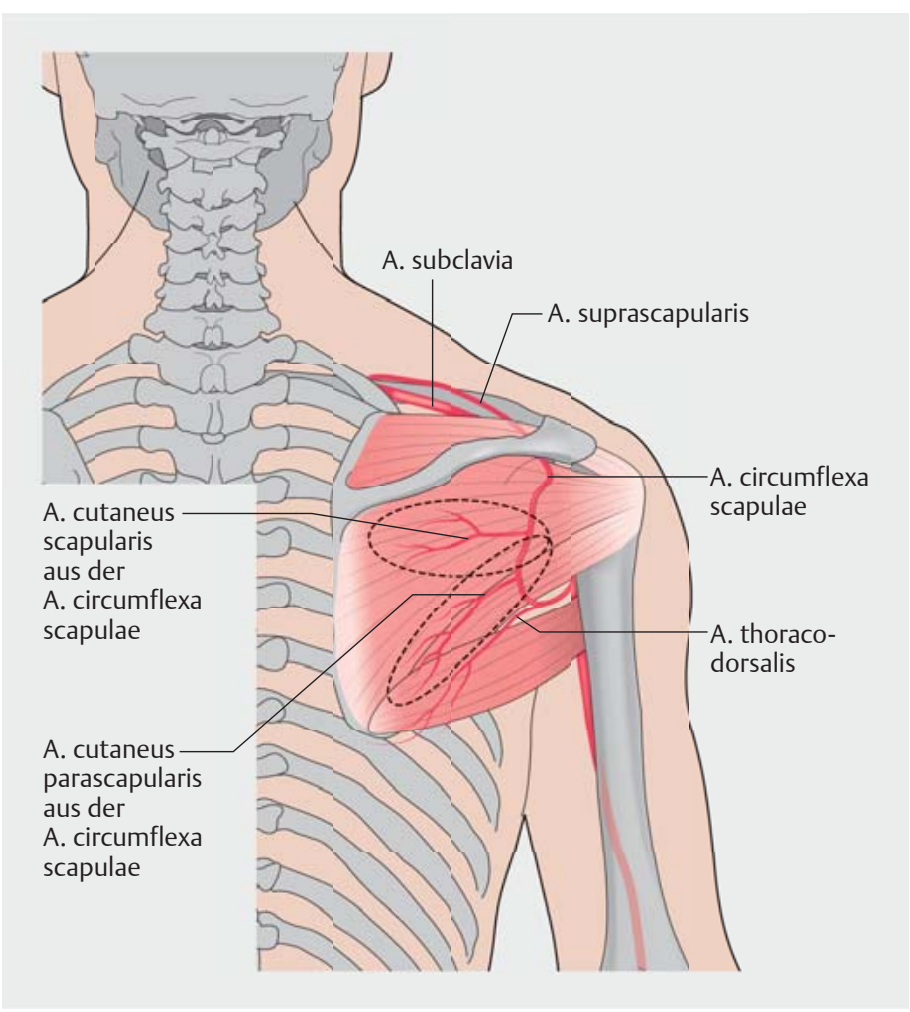

- Abb. 2 Transversal angezeichnet zeigt sich der Skapularlappen gestielt an $\operatorname{der} A$. cutaneus scapularis aus der $A$. circumflexa scapulae, mit dem ein Knochenspan sowohl vom medialen als auch vom lateralen Skapularand gehoben werden kann. Der Paraskapularlappen zeigt sich vertikal angezeichnet und ist an der A. cutaneus parascapularis aus der A. circumflexa scapulae gestielt. Mit dem Paraskapularlappen ist eine Hebung eines Knochenspans nur aus dem lateralen Skapularand möglich. $\operatorname{der}$ A. thoracodorsalis sowie einen Ast der A. dorsalis scapulae der A. subclavia [26]. Der mediale Rand der Skapula wird durch osteofasziokutane Gefäßverbindungen des horizontalen Astes der A. circumflexa scapulae versorgt [27].

Die Präparation des medialen osteokutanen Skapularlappens erfolgt zunächst im Sinne des von dos Santos beschriebenen fasziokutanen Skapularlappens. Dabei wird der Lappen lateral vom M. infraspinatus abgehoben, wobei er im Bereich der Margo medialis an der Skapula anhaftend bleibt. Der Knochenblock kann hier mit einer Länge von 12-14 cm und einer Breite von 2-3 cm gehoben werden [28]. Der laterale osteokutane Skapularlappen wird ebenfalls als osteofasziokutaner Lappen mit dem Skapularlappen oder Paraskapularlappen gehoben.

Die Vorteile des medialen osteokutanen Skapularlappens gegenüber dem lateralen sind die Größe der zu hebenden Hautinsel und die Länge des Pedikels. Weiterhin besteht hier nicht das Risiko einer Schwächung der Rotatorenmanschette, die mit dem lateralen osteokutanen Skapular- oder Paraskapularlappen assoziiert ist. Dem ge- genüber steht jedoch, dass der laterale Teil der Skapula deutlich dicker ist und daher hier ein stärkerer Knochenblock gehoben werden kann [28].

Ähnlich dem vaskularisierten Beckenkammspan findet die gefäßgestielte Knochentransplantation der Skapula aufgrund der konvexen Form hauptsächlich in der Rekonstruktion der Mandibula Verwendung. Im Bereich der oberen Extremität spielt er vornehmlich bei ausgeprägten Weichteildefekten mit kurzen Defektstrecken bis $3 \mathrm{~cm}$ eine Rolle ( $\triangleright$ Abb. $\mathbf{3}$ ).

\section{Kahnbeinpseudarthrosen}

\section{Einleitung}

Mit ca. $80 \%$ ist die Kahnbeinfraktur die häufigste Fraktur der Handwurzelknochen [29]. Dabei stellt das Kahnbein auf mehreren Ebenen eine große Herausforderung in der operativen Frakturversorgung dar. Daher war vor der Entwicklung der intraossären Verschraubung lange Jahre die konservative Therapie mit Ruhigstellungsdauern von bis zu 3 Monaten der Goldstandard [30].

Das Kahnbein spielt eine Schlüsselrolle in der Biomechanik des Carpus und interagiert sowohl mit der proximalen als auch der distalen Handwurzelreihe. Dabei liegt es in einem Winkel von ca. $40^{\circ}\left( \pm 3^{\circ}\right)$ in der Koronarebene sowie $32^{\circ}\left( \pm 5^{\circ}\right)$ in der Sagittalebene verkippt. Knapp 80\% des Kahnbeines sind mit Knorpel überzogen. Die Gefäßversorgung des Kahnbeines erfolgt retrograd, meist direkt aus einem Gefäßabgang der A. radialis [8].

Diese biomechanischen Eigenschaften können eine Dislokation der Frakturenden begünstigen und durch die retrograde Gefäßversorgung kann es zu einer Minderdurchblutung des proximalen Fragmentes kommen. Dabei gilt ab einer Distanz der Frakturenden von 1 mm oder einer Abkippung von mehr als $15^{\circ}$ ein erhöhtes Risiko der Pseudarthrosenentstehung [8]. In der Literatur werden Pseudarthrosenraten von 5-15\% beschrieben [29].

Eine unbehandelte Kahnbeinpseudarthrose ist mit einer progredienten Arthrose des Carpus assoziiert: „scaphoid nonunion advanced collapse“ (SNAC). Dabei kommt es zunächst zu einem vermehrten Gelenkverschleiß zwischen dem Processus styloideus radii und dem proximalen Pol des Kahnbeines. Darauf folgt eine zunehmende Mediokarpalarthrose und schlussendlich die Panarthrose des Carpus [8]. Dabei variieren die in der Literatur angegebenen Arthroseraten von $97 \%$ bereits 5 Jahre nach dem Trauma [31] und 22\% nach weniger als 5 Jahren bzw. 75\% nach 5-9 Jahren [32].

Zur Rekonstruktion des Kahnbeines bei vorliegender Pseudarthrose können zunächst als avaskuläre Rekonstruktionsmöglichkeiten die Frakturenden angefrischt 


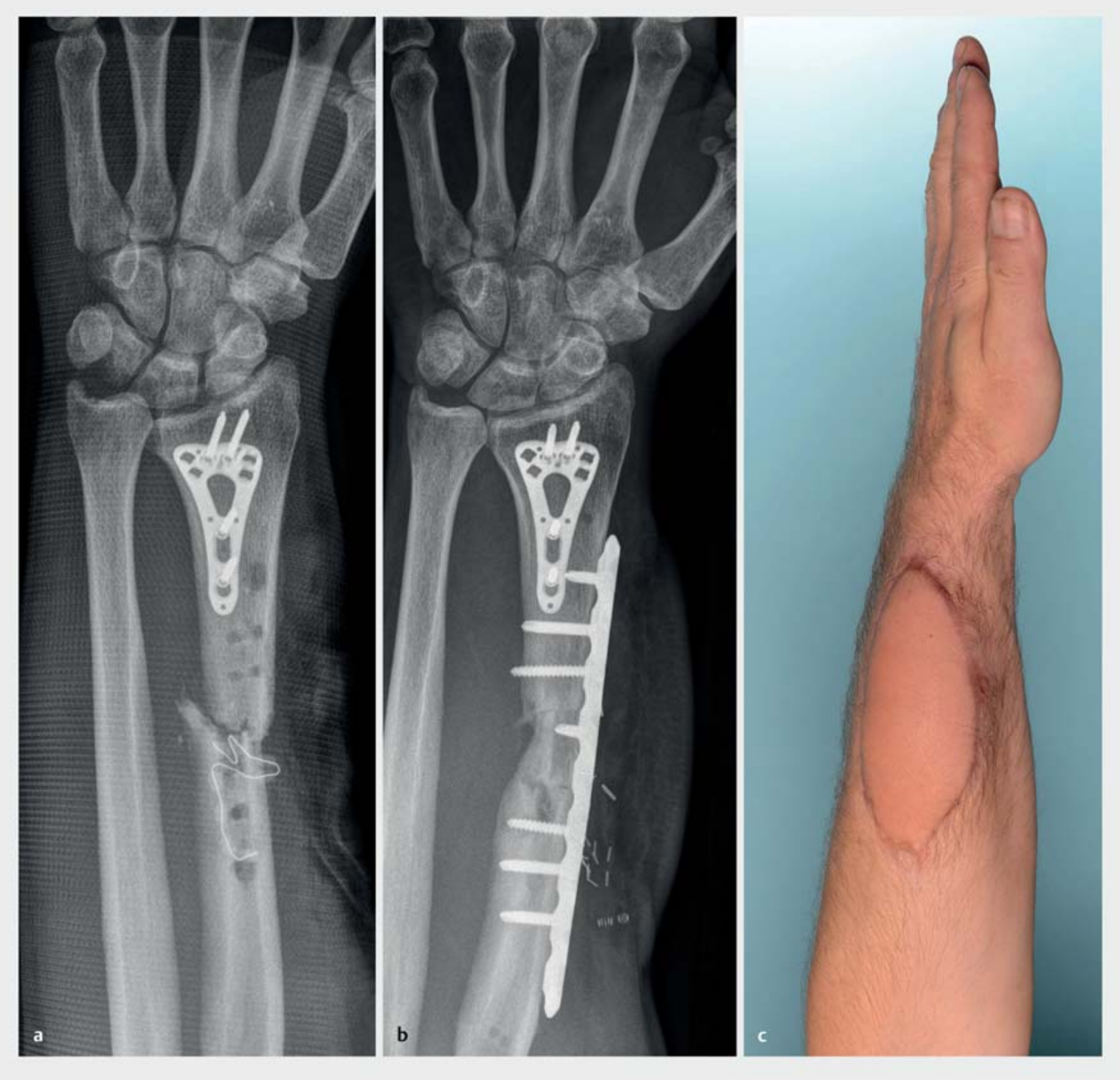

- Abb. 3 a Radiologisches Bild einer Defektpseudarthrose des Radius. b, c Radiologisches und klinisches Ergebnis nach Rekonstruktion eines 2,2 cm langen Radiusdefektes mittels eines osteokutanen Paraskapularlappens.

und der Defekt mit Spongiosa aus dem Beckenkamm oder dem distalen Radius aufgefüllt werden und anschließend mittels einer Kompressionsschraube (z. B. HerbertSchraube [30]) oder seltener Kirschner-Draht-Osteosynthese fixiert werden [29]. Bei einer Achsabknickung nach palmar im Sinne einer „Humpback“-Deformität oder bei einem größeren knöchernen Defekt über $3 \mathrm{~mm}$ empfiehlt sich der Einsatz eines kortikospongiösen Beckenkammspans. Bei persistierender Pseudarthrose oder einem sehr avaskulären proximalen Fragment bieten sich die folgenden vaskularisierten Rekonstruktionsmöglichkeiten an [8].

\section{Der gefäßgestielte Radiusspan}

In der Literatur werden verschiedene gefäßgestielte Radiusspäne zur Therapie der Kahnbeinpseudarthrose beschrieben. Mathoulin und Haerle veröffentlichten 1998 den palmaren vaskularisierten Radiusspan, der mit einer Größe von ca. $1 \mathrm{~cm}^{3}$ gehoben werden kann. Der palmare Anteil des distalen Radius wird dabei durch eine Anastomose zwischen den Ästen der A. ulnaris, A. radialis sowie $\operatorname{der} A$. interossea anterior versorgt. Es konnte hiermit in 9 von 17 Patienten eine Konsolidierung der vorher bestehenden Pseudarthrose innerhalb von 45 Tagen erzielt werden [33].

Am häufigsten verwendet wird jedoch der dorsale gefäßgestielte Radiusspan, der erstmalig 1991 von Zaidem- 

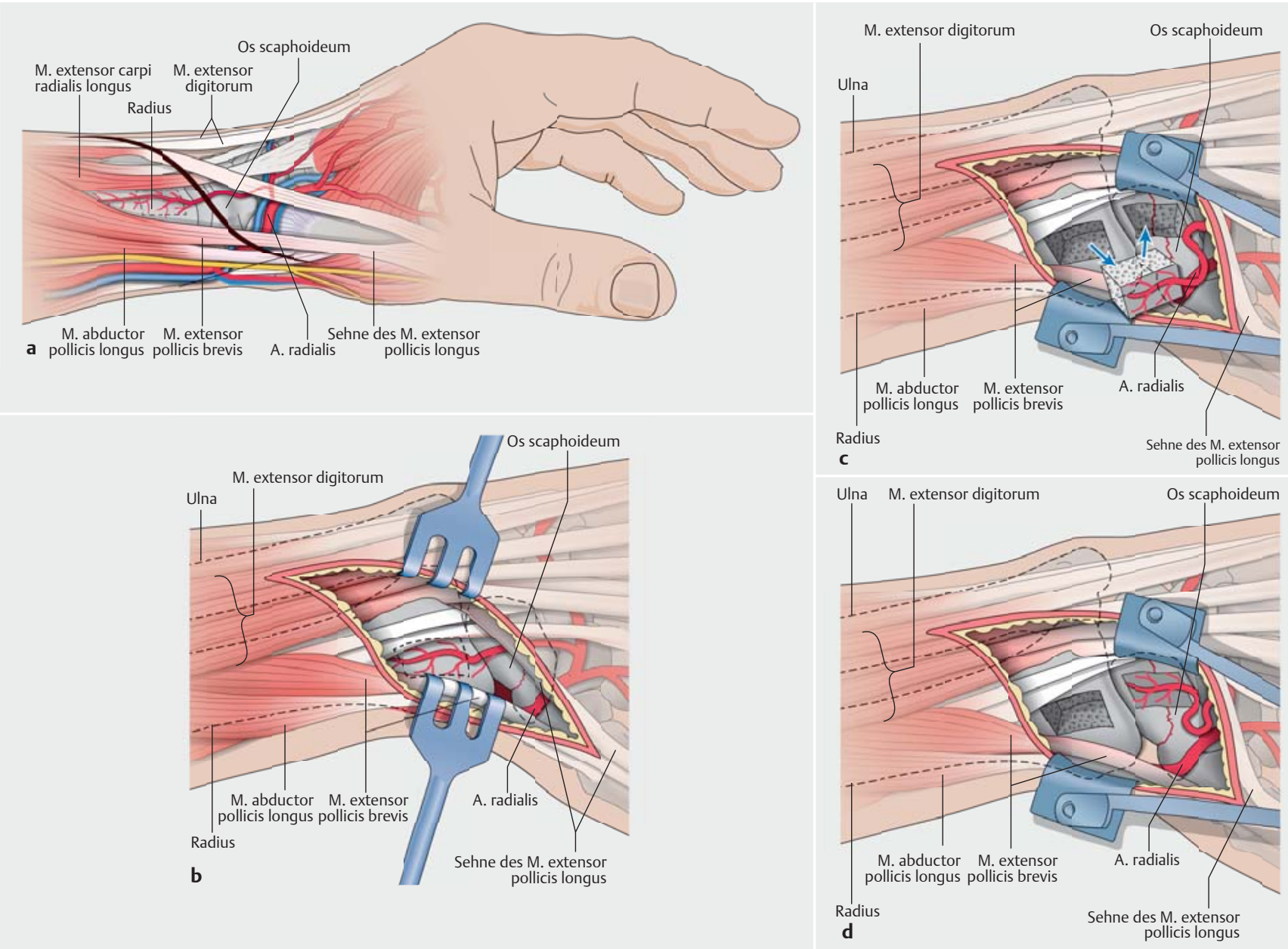

- Abb. 4 a Planen der Schnittführung unter Einbeziehung der anatomischen Strukturen am radiodorsalen Handgelenk. b Darstellung der 1./2. ICSRA auf der Oberfläche des Retinaculum extensorum (RSNR: Ramus superficialis nervi radialis). c Hebung des gestielten Radiusspans mit dem scharfen Meißel. d Der Radiusspan wurde in den Kahnbeindefekt nach Anfrischung der Pseudarthrose eingebracht. Idealerweise erfolgt die Fixation des Spans mit einer kanülierten Herbert-Schraube.

berg et al. beschrieben wurde [34,35]. Die Gefäßversorgung des dorsalen distalen Radius wird über die Lagebeziehung der Gefäße zu den Extensorenfächern definiert [8]. Bei dem von Zaidemberg et al. beschriebenen dorsalen Radiusspan erfolgt die Durchblutung retrograd über Äste der A. radialis. Dabei wird zur Hebung des Spans die 1,2 interkompartimentelle supraretinakuläre Arterie („1,2 intercompartment supraretinacular artery“, 1,2 ICSRA) verwendet [34]. Diese liegt oberhalb des Retinaculum extensorum zwischen dem 1. und 2. Extensorenfach. In diesem Bereich sowie zwischen dem 2. und 3. Strecksehnenfach liegt das Retinaculum dem distalen Radius direkt an. Daher können hier Gefäße in die Kortikalis einsprießen [8] ( $\mathbf{A b b}$. 4).

Die in der Literatur beschriebenen Ergebnisse des Zaidemberg-Spans variieren jedoch sehr stark bei Ausheilungsraten zwischen 27 und $100 \%$ bei einer Nachuntersuchungszeit von 6,5-19 Wochen postoperativ [35].

\section{Der kortikospongiöse bzw. osteochondrale Femurspan}

Der 1991 erstmalig von Sakai et al. beschriebene kortikoperiostale Lappen der medialen Femurkondyle wurde in den Folgejahren insofern modifiziert, dass auch spongiöse Knochenanteile en bloc mitgehoben wurden. Doi et al. publizierten im Jahre 2000 erstmals 10 Fälle, bei denen ein freies mikrovaskuläres Knochentransplantat vom medialen Femurkondylus bei Kahnbeinpseudarthrose verwendet wurde. Die Verwendung der gefäßgestielten medialen Femurkondyle kann bei einer veralteten Kahnbeinpseudarthrose, bei fehlender oder unsicherer Vitalität des proximalen Fragmentes, ausgedehnten Knochendefekten mit z. B. „Humpback“-Deformität, multiplen Voroperationen bzw. Kombinationen dieser Faktoren indiziert sein. Kontraindikationen bilden die zuvor beschriebene fortgeschrittene Arthrose des Carpus (SNAC), Erkrankungen des Gefäßsystems, mangelnde Compliance oder ein reduzierter Allgemeinzustand des Patienten [36]. Auch bei Rauchern oder einer bereits bestehenden Gelenk- 
schädigung der Knie, z. B. im Rahmen vorangegangener Traumata oder einer Adipositas, ist die Indikation kritisch zu stellen. Eine besondere Patientengruppe stellen Kinder dar. Bei noch offenen Wachstumsfugen sollte ebenfalls eine Indikation äußerst kritisch gestellt werden, da insgesamt noch keine ausreichenden Langzeitdaten über die Hebemorbiditäten vorliegen.

Die Gefäßversorgung der medialen Femurkondyle erfolgt über das Rete articulare genus. Dieses Gefäßgeflecht wird aus dem R. articularis der A. genus descendens und dem R. supracondylaris der A. poplitea gebildet. Als Gefäßstiel des Transplantates wird meist der R. articularis verwendet [36]. Der Gefäßstiel der A. genus descendens kann über eine Länge von bis zu $13 \mathrm{~cm}$ bis zum Ursprung aus der A. femoralis superficialis im Adduktorenkanal präpariert werden. Das Gefäßkaliber entspricht dabei etwa $1,5 \mathrm{~mm}$ [37].

Von Bürger et al. wurden im Jahre 200915 Fälle beschrieben, die eine Kahnbeinpseudarthrose im mittleren Drittel mit avitalem proximalen Fragment aufwiesen. Diese wurden im Durchschnitt 128 Wochen nach dem Trauma behandelt. Es zeigte sich 6 Monate postoperativ eine knöcherne Durchbauungsrate von 100\% [36]. Je nach Indikation kann ein vaskularisierter Femurspan auch von lateral gehoben werden, der als kombinierter osteochondraler fasziokutaner Lappen mit vaskularisiertem Sehnentransplantat gehoben werden kann [38].

Im Jahr 2008 wurde von Kälicke und Bürger erstmals die Verwendung eines osteochondralen Femurspans zum Ersatz eines avaskulären proximalen Pols bei Kahnbeinpseudarthrose beschrieben [39]. Zur Rekonstruktion des proximalen Pols wird meist ein Knochenspan von ca. $2 \times 1 \times 1 \mathrm{~cm}$ von der medialen knorpelbedeckten Trochlea gehoben. Dabei entspricht die Länge (proximal/distal) des Spans der Breite des Kahnbeines (radial/ulnar). Die Dicke des Spans (anterior/posterior) entspricht der Länge des zu ersetzenden Defektes am Kahnbein (proximal/distal). Analog dazu entspricht die Breite des Spans (medial/ lateral) der Dicke des Kahnbeines (palmar/dorsal). Die von Knorpel überzogene Fläche des Transplantates artikuliert später mit der Fovea scaphoidea des distalen Radius. Dabei erfolgt die Ausrichtung des Spans so, dass der mediale Rand des Spans dorsal und der laterale Rand palmar im Handgelenk zu liegen kommt. Die Gefäßversorgung des osteochondralen Femurspans erfolgt analog zu derjenigen des kortikospongiösen Femurspans [37].

Im Jahre 2013 veröffentlichten Bürger et al. eine Fallstudie von 16 Patienten, bei denen der Ersatz des proximalen Kahnbeinpols mithilfe des osteochondralen Femurspans durchgeführt wurde. Bei einer mittleren Nachuntersuchungszeit von 14 Monaten postoperativ zeigte sich bei 15 der 16 Patienten eine knöcherne Durchbauung im CT. Dabei konnte bei 12 Patienten eine komplette Schmerz- freiheit erzielt werden. Die prä- und postoperative Handgelenksbeweglichkeit waren gleich [40] (• Abb. 5).

Wie von Kalb 2016 berichtet, konnten in einer ersten Nachuntersuchung von jeweils 30 Patienten eine Heilungsquote von $63 \%$ bei der Verwendung eines kortikospongiösen sowie von $77 \%$ bei Verwendung eines osteochondralen Femurspans, bei einer durchschnittlichen Nachbeobachtungszeit von 12 bzw. 14 Monaten, gezeigt werden [41]. Bei 4 Patienten dieser ersten Nachuntersuchungsreihe zeigte sich eine Ossifikation des Gefäßstiels, jedoch ohne Revisionsnotwendigkeit. In einem Fall kam es 3 Monate nach Operation im Rahmen eines Traumas zu einer Oberschenkelfraktur [42].

\section{Der vaskularisierte kortikospongiöse Beckenkammspan}

Die Transplantation eines vaskularisierten Beckenkammspans wurde erstmalig im Jahre 1978 von Taylor et al. bzw. 1979 von Sanders et al. beschrieben [43, 44].

Die Blutversorgung des Beckenkamms erfolgt über die A. circumflexa ilium superficialis („superficial circumflex iliac artery“, SCIA) sowie die entscheidendere A. circumflexa ilium profunda („deep circumflex iliac artery“, $D C I A)$. Die Länge des Gefäßstiels der „DCIA“ variiert von $7-10 \mathrm{~cm}$ bis zu $15-30 \mathrm{~cm}$ bei einem Durchmesser von ca. $2,8 \mathrm{~mm}$ [8].

Neben dem kortikospongiösen Span bestehen hier auch die Möglichkeiten, einen osteokutanen oder osteomuskulären Lappen, unter Mitnahme des M. obliquus internus, zu heben. So kann ein konvexer Span von maximal ca. $10 \mathrm{~cm}$ Länge gehoben werden, sodass dieser Knochentransfer eine untergeordnete Rolle bei Defekten der geraden, langen Röhrenknochen spielt und vornehmlich bei großen Weichteildefekten oder kleineren Knochendefekten an der Hand bzw. dem Handgelenk verwendet wird [8]. Einen größeren Stellenwert hat der vaskularisierte Beckenkamm aufgrund seiner gebogenen Form in der Rekonstruktion der Mandibula.

\section{Fazit}

Generell lässt sich sagen, dass die Auswahl des geeigneten operativen Verfahrens zur Behandlung einer Pseudarthrose nach dem zugrunde liegenden Pathomechanismus getroffen werden sollte. Ist aufgrund einer unzureichenden Stabilisierung der Fraktur die Knochenbruchheilung ausgeblieben, sollte eine (erneute) Osteosynthese durchgeführt werden. Eine Defektpseudarthrose kann mithilfe eines Knochenspans oder einer Spongiosaplastik aufgefüllt und osteogene Zellen in diesen Bereich eingebracht werden. Dabei kann eine Knochenminderdurchblutung durch die Verwendung eines vaskulären Knochentransplantates verbessert werden. Die Auswahl des geeigneten Verfahrens sollte dabei anhand der Größe 

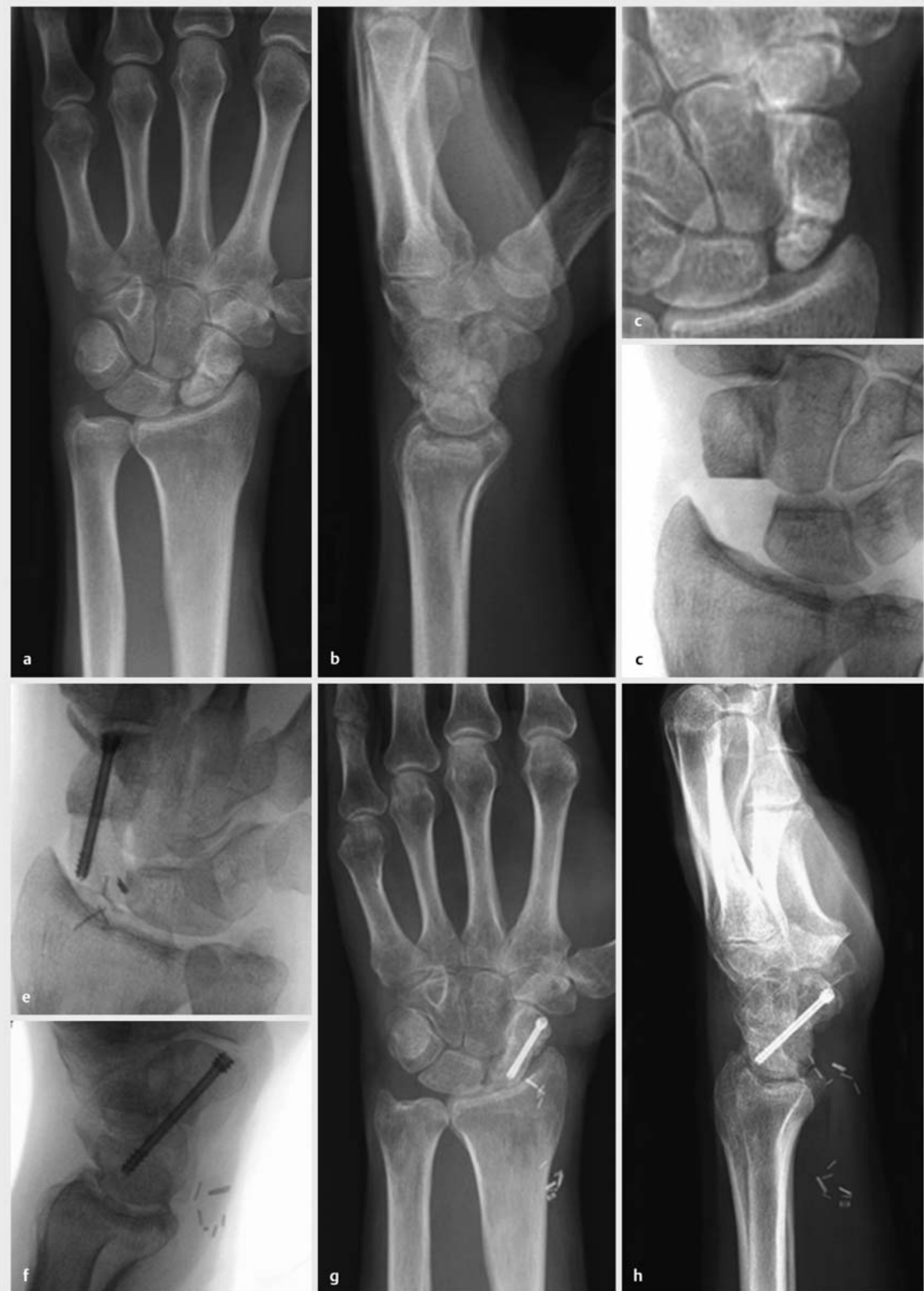

c
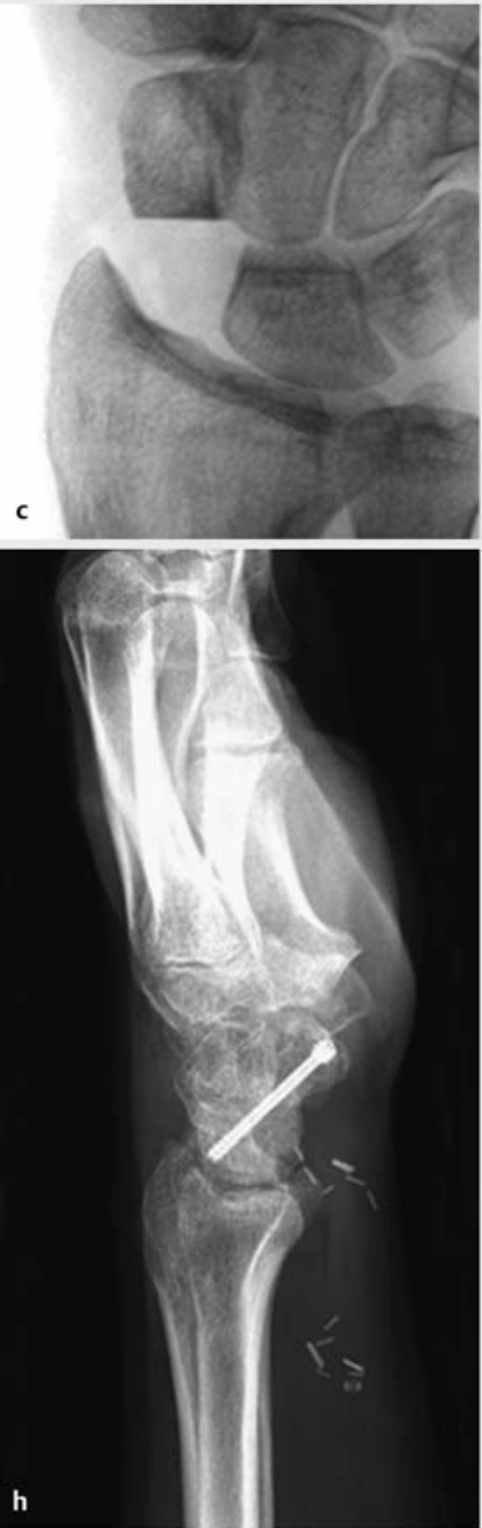

- Abb. 5 a-c Radiologisches Bild einer Kahnbeinpseudarthrose des linken Handgelenkes bei einem 47-jährigen Patienten. d Intraoperative Darstellung nach Resektion der Pseudarthrose sowie des proximalen Pols. e, $\mathbf{f}$ Intraoperative Darstellung nach Ersatz des proximalen Kahnbeinpols mithilfe des vaskularisierten Femurspans und Herbert-Schrauben-Osteosynthese. g, h Radiologisches Bild ca. 5 Monate nach proximalem Polersatz mittels medialer vaskularisierter Femurkondyle. 
des Knochendefektes sowie der begleitenden Weichteildefekte getroffen werden. Weiterhin ist zu beachten, dass eine gewisse mikrochirurgische Expertise sowie ein guter Allgemeinzustand des Patienten bei allen Verfahren Voraussetzung sind.

Bei einer langstreckigen Defektpseudarthrose des Unterarmes stellt die freie gefäßgestielte Fibulatransplantation die Methode der Wahl dar. Dabei können Defekte von bis zu $28 \mathrm{~cm}$ überbrückt werden. Der kortikoperiostale Lappen der medialen Femurkondyle bietet mit seinen osteogenen Potenzen eine gute Alternative bei kleinen Defekten oder anatomisch anspruchsvollen Empfängerregionen. Trotz bislang guter Ergebnisse wird dieser Lappen noch nicht standardmäßig verwendet. Hier bleiben größere Fallzahlen und weitere Publikationen abzuwarten.

Der vaskularisierte Beckenkammspan sowie die gefäßgestielte Transplantation der medialen oder lateralen Skapula finden hauptsächlich bei kleinen knöchernen oder ausgeprägten Weichteildefekten Verwendung. Bei einer größeren zu überbrückenden knöchernen Defektstrecke müssten bei Verwendung des Beckenkamms mehrere Osteotomien durchgeführt werden, um die Konvexität auszugleichen. Einen größeren Stellenwert als in der Chirurgie der oberen Extremität haben beide Verfahren in der Kopf- und Halschirurgie im Rahmen der Mandibularekonstruktion.

Bei einer persistierenden Kahnbeinpseudarthrose bieten sich als vaskularisierte Rekonstruktionsmöglichkeiten sowohl der gefäßgestielte dorsale Radiusspan als auch der freie kortikospongiöse mediale Femurspan an. Bei einem avaskulären proximalen Pol bietet der osteochondrale Femurspan die Möglichkeit des Polersatzes. Im Gegensatz zu dem Einsatz eines osteochondralen bzw. kortikospongiösen Spans der medialen Femurkondyle ist der beschriebene Zaidemberg-Span aus dem distalen Radius mit deutlich größeren publizierten Fallzahlen jedoch das bereits besser etablierte Verfahren. Weiterhin ist die anspruchsvolle Operationstechnik bei Verwendung der Femurkondyle zu beachten, bei der das Transplantat exakt in den Defekt eingepasst werden muss, ohne den Gefäßstiel und das Periost zu beschädigen. Die mikrovaskulär angeschlossenen Femurknochentransplantate bieten eine Erweiterung der therapeutischen Behandlungsmöglichkeiten insbesondere therapierefraktärer Kahnbeinpseudarthrosen. Bei jedoch noch kleinen Fallzahlen, fehlenden Langzeitergebnissen sowie einer unzureichenden Auswertung von Komplikationen und kritischer Analyse des Hebedefektes sollte die Indikation kritisch gestellt werden.

\section{Autorinnen/Autoren}

\section{Saskia Carolin Evers}

Assistenzärztin, Klinik für Hand-, Plastische, Rekonstruktive und Verbrennungschirurgie an der Eberhard-Karls-Universität Tübingen

\section{Adrien Daigeler}

Prof. Dr. med., Direktor der Klinik für Hand-, Plastische, Rekonstruktive und Verbrennungschirurgie, Klinik für Hand-, Plastische, Rekonstruktive und Verbrennungschirurgie an der Eberhard-Karls-Universität Tübingen

\section{Theodora Wahler}

PD Dr. med., Leitende Oberärztin, Klinik für Hand-, Plastische, Rekonstruktive und Verbrennungschirurgie an der EberhardKarls-Universität Tübingen

Korrespondenzadresse

\section{Saskia Carolin Evers}

Klinik für Hand-, Plastische, Rekonstruktive und

Verbrennungschirurgie

BGU Tübingen

Schnarrenbergstraße 95

72076 Tübingen

severs@bgu-tuebingen.de

Literatur

[1] Ruchholtz S, Wirtz DC, Hrsg. Orthopädie und Unfallchirurgie essentials. Stuttgart: Thieme; 2010

[2] Schmidmaier G, Moghaddam A. Pseudarthrosen langer Röhrenknochen. Z Orthop Unfall 2015; 153: 659-676

[3] Pillukat T, Fuhrmann R, Windolf J et al. Die palmare winkelstabile Plattenosteosynthese bei Extensionsfrakturen des distalen Radius. Oper Orthop Traumatol 2016; 28: 47-64

[4] Walz M, Kolbow B, Möllenhoff G. Distale Ulnafraktur als Begleitverletzung des körperfernen Speichenbruchs: Minimalinvasive Versorgung mittels elastisch-stabiler intramedullärer Nagelung (ESIN). Unfallchirurg 2006; 109: 1058-1063

[5] Arora R, Lutz M, Zimmermann R et al. Grenzen der palmaren winkelstabilen Plattenosteosynthese bei instabilen distalen Radiusfrakturen. Handchir Mikrochir Plast Chir 2007; 39: 3441

[6] Müller-Mai CM, Mielke E. Unterarmschaft. In: Müller-Mai C, Ekkernkamp A, Hrsg. Frakturen auf einen Blick. Berlin, Heidelberg: Springer; 2015: 81-98

[7] Marcheix P-S, Delclaux S, Ehlinger M et al. Pre- and postoperative complications of adult forearm fractures treated with plate fixation. Orthop Traumatol Surg Res 2016; 102: 781-784

[8] Wolfe SW, Hotchkiss RN, Pederson WC, Kozin SH, Cohen MS, eds. Green's operative Hand Surgery. Seventh edition. Philadelphia, PA: Elsevier; 2017

[9] Huntington TW. Case of bone transference. Ann Surg 1905; 41: 249-251

[10] Eisenschenk A, Lehnert M, Weber U. Die freie, gefäßgestielte Fibulatransplantation zur Überbrückung von Knochendefekten. Oper Orthop Traumatol 1994; 6: 107-118 
[11] Gilbert A. Free vascularized bone grafts. Int Surg 1981; 66: 27-31

[12] Chen ZW, Yan W. The study and clinical application of the osteocutaneous flap of fibula. Microsurgery 1983; 4: 11-16

[13] Pederson WC, Person DW. Long bone reconstruction with vascularized bone grafts. Orthop Clin North Am 2007; 38: 23-35

[14] Jones NF, Swartz WM, Mears DC et al. The "double barrel" free vascularized fibular bone graft. Plast Reconstr Surg 1988; 81: 378-385

[15] Innocenti M, Abed YY, Beltrami G et al. Biological reconstruction after resection of bone tumors of the proximal tibia using allograft shell and intramedullary free vascularized fibular graft: long-term results. Microsurgery 2009; 29: 361-372

[16] McKee NH, Haw P, Vettese T. Anatomic study of the nutrient foramen in the shaft of the fibula. Clin Orthop Relat Res 1984; (184): 141-144

[17] Liu S, Tao S, Tan J et al. Long-term follow-up of fibular graft for the reconstruction of bone defects: Medicine (Baltimore) 2018; 97: e12605

[18] Soucacos PN, Dailiana Z, Beris AE et al. Vascularised bone grafts for the management of non-union. Injury 2006; 37 (Suppl. 1): S41-S50

[19] Sakai K, Doi K, Kawai S. Free vascularized thin corticoperiosteal graft. Plast Reconstr Surg 1991; 87: 290-298

[20] Del Piñal F, García-Bernal F], Regalado J et al. Vascularised corticoperiosteal grafts from the medial femoral condyle for difficult non-unions of the upper limb. J Hand Surg Eur Vol 2007; 32: 135-142

[21] De Smet L. Treatment of non-union of forearm bones with a free vascularised corticoperiosteal flap from the medial femoral condyle. Acta Orthop Belg 2009; 75: 611-615

[22] Choudry UH, Bakri K, Moran SL et al. The vascularized medial femoral condyle periosteal bone flap for the treatment of recalcitrant bony nonunions. Ann Plast Surg 2008; 60: 174-180

[23] Kaminski A, Bürger $H$, Müller E]. Free vascularised corticoperiosteal bone flaps in the treatment of non-union of long bones: an ignored opportunity? Acta Orthop Belg 2008; 74: 235-239

[24] Stokes R, Whetzel TP, Stevenson TR. Three-dimensional reconstruction of the below-knee amputation stump: use of the combined scapular/parascapular flap. Plast Reconstr Surg 1994; 94: 732-736

[25] Koshima I, Soeda S. Repair of a wide defect of the lower leg with the combined scapular and parascapular flap. Br J Plast Surg 1985; 38: 518-521

[26] Sundine MJ, Sharobaro VI, Ljubic I et al. Inferior angle of the scapula as a vascularized bone graft: an anatomic study. J Reconstr Microsurg 2000; 16: 207-211

[27] Sauerbier M, Erdmann D, Bickert B et al. [Defect coverage of the hand and forearm with a free scapula-parascapula flap]. Handchir Mikrochir Plast Chir 2001; 33: 20-25

[28] Thoma A, Archibald S, Payk I et al. The free medial scapular osteofasciocutaneous flap for head and neck reconstruction. $\mathrm{Br}$ J Plast Surg 1991; 44: 477-482

[29] Mehling I, Sauerbier M. Skaphoidfrakturen und Skaphoidpseudarthrosen. Z Orthop Unfall 2013; 151: 639-660
[30] Krimmer H, Schmitt R, Herbert T. Kahnbeinfrakturen - Diagnostik, Klassifikation und Therapie. Unfallchirurg 2000; 103: 812-819

[31] Mack GR, Bosse M], Gelberman RH et al. The natural history of scaphoid non-union. J Bone Joint Surg Am 1984; 66: 504-509

[32] Inoue G, Sakuma M. The natural history of scaphoid nonunion. Radiographical and clinical analysis in 102 cases. Arch Orthop Trauma Surg 1996; 115: 1-4

[33] Mathoulin C, Haerle M. Vascularized bone graft from the palmar carpal artery for treatment of scaphoid nonunion. J Hand Surg $\mathrm{Br}$ 1998; 23: 318-323

[34] Zaidemberg C, Siebert JW, Angrigiani C. A new vascularized bone graft for scaphoid nonunion. J Hand Surg Am 1991; 16 : 474-478

[35] Henry M. Scaphoid nonunion: what is the role of the Zaidemberg 1,2 intercompartmental supraretinacular arterial flap? I Hand Surg Eur Vol 2018; 43: 41-47

[36] Bürger H, Gaggl A], Kukutschki W et al. Das freie mikrovaskuläre Knochentransplantat vom medialen Femurkondylus zur Behandlung der Kahnbeinpseudarthrose. Oper Orthop Traumatol 2009; 21: 396-404

[37] Higgins JP, Bürger HK. Medial femoral trochlea osteochondra flap. Clin Plast Surg 2017; 44: 257-265

[38] Parvizi D, Vasilyeva A, Wurzer P et al. Anatomy of the vascularized lateral femoral condyle flap. Plast Reconstr Surg 2016; 137: $1024 \mathrm{e}-1032 \mathrm{e}$

[39] Kälicke T, Bürger H, Müller E]. Freies gefäßgestieltes KnorpelKnochen-Transplantat bei avaskulärer Nekrose des proximalen Kahnbeinpols: Beschreibung eines neuartigen Operationsverfahrens. Unfallchirurg 2008; 111: 201-205

[40] Bürger HK, Windhofer C, Gaggl A] et al. Vascularized media femoral trochlea osteocartilaginous flap reconstruction of proximal pole scaphoid nonunions. J Hand Surg 2013; 38: 690-700

[41] Kalb K. Freies gefäßgestieltes Femurknochentransplantat be Skaphoidpseudarthrose: Indikation und Technik. Orthopäde 2016; 45: 966-973

[42] Kalb K, Blanarsch B, Prommersberger K-J. Erste Erfahrungen mit dem freien mikrovaskulär angeschlossenen Femurspan zur Skaphoidrekonstruktion. 53. Kongress der Deutschen Gesellschaft für Handchirurgie 2012. Im Internet: http:// www.egms.de/en/meetings/dgh2012/12dgh33.shtml; Stand: 23.06.2019

[43] Taylor GI, Townsend P, Corlett R. Superiority of the deep circumflex iliac vessels as the supply for free groin flaps. Clinical work. Plast Reconstr Surg 1979; 64: 745-759

[44] Sanders R, Mayou BJ. A new vascularized bone graft transferred by microvascular anastomosis as a free flap. $\mathrm{Br}$ I Surg 1979; 66: 787-788

Bibliografie

DOI https://doi.org/10.1055/a-0899-5345

OP-JOURNAL 2019; 35: 231-240 ㄷ Georg Thieme Verlag KC Stuttgart · New York ISSN 0178-1715 